\title{
The 12 Years Preceding Mild Cognitive Impairment Due to Alzheimer's Disease: The Temporal Emergence of Cognitive Decline
}

\author{
Panagiota Mistridis, ${ }^{\mathrm{a}, \mathrm{b}}$, Sabine Krumm ${ }^{\mathrm{a}, \mathrm{b}}$, Andreas U. Monsch ${ }^{\mathrm{a}, \mathrm{b}, *}$, Manfred Berres $^{\mathrm{c}}$ \\ and Kirsten I. Taylor ${ }^{\mathrm{a}, \mathrm{b}, \mathrm{d}, 1}$ \\ ${ }^{a}$ Memory Clinic, University Center for Medicine of Aging Basel, Felix Platter Hospital, Basel, Switzerland \\ ${ }^{\mathrm{b}}$ University of Basel, Basel, Switzerland \\ ${ }^{\mathrm{c}}$ Department of Mathematics and Technology, University of Applied Sciences Koblenz, Koblenz, Germany \\ ${ }^{\mathrm{d}}$ Centre for Speech, Language and the Brain, Department of Experimental Psychology, University of Cambridge, \\ Cambridge, $U K$
}

Handling Associate Editor: Thomas Benke

\begin{abstract}
.
Background: The identification of the type and sequence of cognitive decline in preclinical mild cognitive impairment (MCI) prior to Alzheimer's disease (AD) is crucial for understanding AD pathogenesis and implementing therapeutic interventions. Objective: To model the longitudinal courses of different neuropsychological functions in MCI due to AD.

Methods: We investigated the prodromal phase of MCI over a 12-year period in 27 initially healthy participants with subsequent MCI preceding $\mathrm{AD}$ (NC-MCI) and 60 demographically matched healthy individuals (NC-NC). The longitudinal courses of cognitive performance (verbal and visual episodic memory, semantic memory, executive functioning, constructional praxis, psychomotor speed, language, and informant-based reports) were analyzed with linear mixed effects models.

Results: The sequence with which different cognitive functions declined in the NC-MCI relative to the NC-NC group began with verbal memory and savings performance approximately eight years, and verbal episodic learning, visual memory, and semantic memory (animal fluency) circa four years prior to the MCI diagnosis. Executive functioning, psychomotor speed, and informant-based reports of the NC-MCI group declined approximately two years preceding the MCI diagnosis.

Conclusions: Measurable neuropsychological deterioration occurs up to approximately eight years preceding MCI due to AD.
\end{abstract}

Keywords: Alzheimer's disease, cognitive decline, neuropsychology, linear mixed effects models, longitudinal course, mild cognitive impairment, prodromal

\footnotetext{
${ }^{1}$ Current address: Neuroscience, Ophtalmology, and Rare Diseases (NORD), Roche Pharma Research and Early Development, Roche Innovation Center Basel, F. Hoffmann-La Roche, Ltd, Basel, Switzerland.

*Correspondence to: Andreas U. Monsch, Memory Clinic, University Center for Medicine of Aging Basel, Felix Platter Hospital, Schanzenstrasse 55, 4031 Basel, Switzerland. Tel.: +41 61265 31 93; Fax: +41 6126537 94; E-mail: Andreas.Monsch@unibas.ch.
}

\section{INTRODUCTION}

An understanding of the development of neuropsychological symptoms preceding mild cognitive impairment (MCI) prior to Alzheimer's disease (AD) is crucial for understanding the pathogenesis of AD. This very early point on the $\mathrm{AD}$ dementia continuum when cognitive impairments are not yet manifested in daily life offers unique and potentially optimal opportunities 
for disease-modifying interventions [1]. However, few studies examined the longitudinal course of cognitive changes prior to $\mathrm{MCI}$, and hence, little is currently known about the evolution of cognitive decline in preclinical MCI. While future MCI patients' cognitive performance in the preclinical MCI stage is still within normal limits according to diagnostic criteria, at some point in time, their neuropsychological functioning necessarily begins to decline, diverging from the performance of individuals who remain cognitively healthy. The present study addresses these issues by modeling the longitudinal courses of performance on a comprehensive neuropsychological test battery administered biennially to originally healthy older individuals, some of whom developed MCI prior to $\mathrm{AD}$, over a 12-year observation period.

Verbal and visual episodic memory appear to be the first and most prominently affected cognitive functions in the earliest stages of preclinical MCI [2, 3] and AD ([4-6]; for an overview see [7]). For example, longitudinal studies focusing on specific time points of cognitive changes (i.e., change points models) in preclinical $\mathrm{AD}$ reported declines in episodic immediate or delayed free recall measures from seven to 10 years prior to the diagnosis $[5,8]$. These findings are consistent with the earliest cortical deposition of neurofibrillary pathology related to neural and thus neuropsychological dysfunction in the anteromedial temporal lobe, i.e., the entorhinal cortex and hippocampus, structures critical for episodic memory functioning [9-11].

The medial perirhinal cortex is affected by neurofibrillary pathology prior to the entorhinal cortex and hippocampus [10,12], a structure associated with the processing of semantic object memories [13]. While several studies examining pre-MCI and pre-AD patients found that semantic memory as measured by the semantic fluency task declined first or concurrently with episodic memory $[14,15]$, the majority of studies report that episodic memory declined prior to semantic memory in the earliest stages of AD (e.g., [5, 6]).

Other research suggests that cognitive decline in the preclinical stages of MCI and AD is more global in nature and includes concurrent changes in different cognitive domains including memory. Specifically, a decline in executive function, psychomotor/perceptual speed, attentional processes, or visuospatial abilities (see [16]) are hypothesized to be affected concurrently or shortly after episodic and semantic memory.

The earliest changes in cognitive functioning in future dementia patients may be perceived by intimates who are familiar with the future patient's current and premorbid level of cognitive functioning [17-19]. Isella et al. [19] examined the power of a common, standardized informant questionnaire assessing perceived changes in daily cognitive functioning, the Informant Questionnaire on Cognitive Decline in the Elderly (IQCODE; [20, 21]), to predict progression from MCI to future dementia over a 2-year time frame. A logistic regression analysis indicated that the IQCODE indeed predicted future dementia with a correct classification rate of $81 \%$. These findings suggest that informantbased reports may provide valuable information on the earliest cognitive changes in future $\mathrm{MCI}$ and $\mathrm{AD}$ patients.

To our knowledge few studies have investigated individuals' cognitive functioning in the time period preceding the MCI diagnosis. As expected, these studies indicate that cognitive performance declines several years before the MCI diagnosis [15, 22, 23], similar to the findings with pre-AD patients, with varying profiles of longitudinal cognitive declines reported [15, 23]. Moreover, few studies determined the putative cause of MCI in their sample. A study by Howieson et al. [15] examined the 8-year longitudinal courses of episodic verbal memory, semantic memory (semantic fluency task, animals), and visuospatial abilities in 68 future MCI patients and 88 healthy controls. They found that although a decline in episodic memory was apparent in future MCI patients approximately three years prior to the MCI diagnosis, it was preceded by changes in semantic memory and visuospatial abilities by circa one year (see also [14]). An additional analysis including only those participants with future AD revealed even earlier change points in these cognitive measures. Such studies highlight the importance of determining the subsequent dementia diagnosis when investigating the evolution of cognitive decline in preclinical MCI.

The purpose of this study was to model the longitudinal courses of multiple cognitive functions in the 12 years preceding the diagnosis of $\mathrm{MCI}$ in patients who all went on to develop AD. Specifically, we aimed to determine the time points preceding MCI diagnosis at which each neuropsychological and clinical variable declined from the course of a maximally large, demographically matched control group.

\section{MATERIALS AND METHODS}

\section{Participants}

Data from participants of the longitudinal BASEL project (BAsel Study on the ELderly) were analyzed. Baseline testing was conducted between 1997 and 
2001 and included a thorough clinical examination to ensure participants' healthy cognitive status (medical history questionnaire, neuropsychological evaluation, and assessment of depression). Comprehensive neuropsychological follow-up evaluations were conducted every two years until the end of 2013 [24]. Since baseline, 27 participants progressed to MCI (NC-MCI; amnestic MCI $n=26$ [9 single domain; 17 multiple domains]; non-amnestic MCI, multiple domains $n=1$ ) and then on to AD. The mean time between baseline testing and the MCI diagnosis was 7.76 years $( \pm 3.47$ standard deviations [SD]; range 2.11-14.85 years), and between MCI and AD diagnoses 2.32 years $( \pm 1.82 S D$; range $0.5-8.3$ years). MCI was diagnosed by experienced neuropsychologists and neurologists. The whole dataset per individual was available to the clinicians during the diagnostic process. MCI diagnoses were made when: (1) at least two neuropsychological test variables were abnormal (cut-off: $z \leq-1.28$ ) and had declined from previous performance levels, and (2) activities of daily living were intact as assessed with a semi-structured interview with the participants, and (3a) informants observed a decline in cognitive functioning in the preceding two years and/or (3b) the participant expressed concerns about cognitive dysfunction $[25,26]$. If inconsistencies between selfand informant-reports were detected, informants were contacted by telephone to obtain more detailed information. These criteria correspond to the core clinical diagnostic criteria for MCI due to AD proposed by Albert et al. [27]. We note that the present study participants did not have traditional biomarker data available (e.g., MRI, PET, CSF measures) at the time of MCI diagnosis; however, these data are not required to diagnose MCI due to AD according to Albert et al. core clinical criteria (cf. [27], p. 278). Participants diagnosed with MCI remained in the BASEL study. If there were any self- or informant reported concerns about further cognitive deterioration or if objective cognitive decline was evident during follow-up evaluations, participants were referred in house to the Basel Memory Clinic for a thorough interdisciplinary examination including additional neuropsychological testing, MRI, medical and neurological examinations, and blood and serum analyses. AD was diagnosed in a consensus conference according to the Diagnostic and Statistical Manual of Mental Disorders (4th edition; DSM-IV; [28]) and the National Institute of Neurological and Communicative Disorders and Stroke and the Alzheimer's Disease and Related Disorders Association (NINCDS-ADRDA) criteria [29]. If a Basel Memory Clinic examination was not possible, diagnoses were made by experienced external neurologists ( $n=1$ diagnosis) or the participants' general practitioners in consultation with the Basel Memory Clinic ( $n=2$ diagnoses made by two independent practitioners) according to the DSM-IV [28].

Data from sixty cognitively healthy BASEL participants (NC-NC) who remained healthy throughout the entire observation period $($ mean $=11.53$ years $[ \pm 3.68$ $S D$; range 2.61-15.53 years]) and with comparable demographic statuses were also analyzed (see Table 1; all $p$-values $\geq 0.56$ ). Since individuals in the NC-MCI group had different observation periods (i.e., differing lengths of time between baseline testing and the diagnosis of $\mathrm{MCI}$ ), the largest possible subsample of NC-NC individuals was selected from the entire $\mathrm{NC}-\mathrm{NC}$ sample for each visit to ensure demographic comparability between NC-NC and NC-MCI individuals (cf. Table 1). A summary of the participants' APOE status and health characteristics is provided in Supplementary Table 1 . This study was approved by the ethics committee of both Basel and written informed consent was obtained from each participant.

\section{Neuropsychological measures}

At each visit, all participants were administered the same version of the Consortium to Establish a Registry for Alzheimer's Disease - Neuropsychological Assessment Battery (CERAD-NAB; [31]), a comprehensive neuropsychological examination (see Table 2). Only data from tests administered to each participant at each visit were analyzed. All raw scores were transformed into demographically adjusted (age, gender, and education) $z$-scores according to a normative sample [32, 33]. The sample investigated in our study is a subsample of this normative sample (7\% of the normative sample). Some participants were administered the California Verbal Learning Test (CVLT; [34]) instead of the CERAD-NAB word list test: 23/348 NC-NC visits (14 NC-NC participants), and 9/121 NC-MCI visits (8 NC-MCI participants). These CVLT encoding, delayed free recall, and discriminability scores were transformed into corresponding CERAD-NAB word list scores (see [35]). The Trail Making Test part A (TMT-A) and B (TMT-B) datasets had missing values due to premature test termination (e.g., lack of concept understanding) or time-outs (TMT-A: $180 \mathrm{~s}$, TMT-B: 300 s) (TMT-A: 0/348 NC-NC and 3/121 NCMCI visits (3 NC-MCI participants); TMT-B: 8/348 NC-NC and 8/121 NC-MCI visits (4 NC-NC and 5 NCMCI participants)). Missing values were replaced with the minimum score and adjusted using a malus (i.e., 


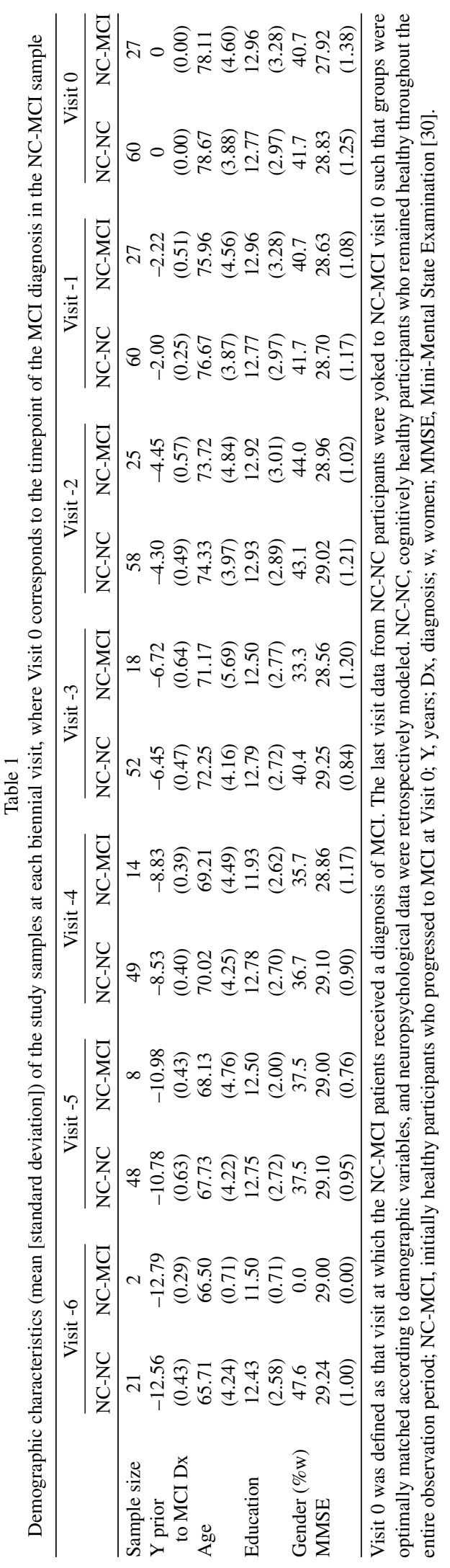

estimated penalty) procedure based on CERAD-NAB total scores in an independent sample of 550 dementia patients (TMT-A $=-0.4$; TMT-B $=-0.7$; see [36] for details).

Participants' informants completed the 16-item IQCODE [20,21] comparing participants' current cognitive functioning to 2 years ago on a five-point scale ( 1 = much improved; $2=$ a bit improved; $3=$ not much change $;=$ a bit worse $;=$ much worse). Thus, a mean score of 3 represents no perceived cognitive decline, and a mean score of 3.3 or above indicates significant perceived cognitive decline [21].

Thus, the present analyses compared the biennial neuropsychological performance of 27 participants who obtained a diagnosis of MCI (and later AD) with 60 participants who remained cognitively healthy. The timepoint of the diagnosis of MCI (for the MCI group) or end of study (NC group) served as the endpoint ("visit 0 "). The goal of the present study was to examine changes in neuropsychological functioning in the pre-MCI phase.

\section{Statistical analyses}

The longitudinal courses of NC-NC and NC-MCI performance on each neuropsychological measure were modeled with linear mixed effects models. These account for correlations between repeated measures from the same participant and permit the analysis of longitudinal data with varying observation periods. Participants were modeled as random effects and diagnostic group, a cubic spline of time until MCI diagnosis (years) and the interaction of group with the cubic spline as fixed effects. The cubic spline is a piecewise cubic function with one knot. It permits a sufficiently flexible model and one adapted to the estimated courses of the behavioral data. Cubic splines are commonly fitted to data if simple linear, quadratic, or cubic functions are inadequate because of the existence of local minima and maxima or because portions of the function are nearly linear or even constant in some intervals. The knot was placed at an a priori determined timepoint such that comparable amounts of data were available for modeling prior to and after the knot, i.e., at -4.65 years before Visit 0 , where Visit 0 corresponds to the timepoint of the MCI diagnosis in the NC-MCI group. One knot was considered to be sufficient to model the nonlinear relationship with at most one maximum or minimum. The interaction of diagnostic group with each term of the cubic spline allows separate models to be estimated for each group. Estimated marginal means (i.e., group means computed from the fixed effects of 
Table 2

Description of the neuropsychological variables used in the present study

\begin{tabular}{|c|c|c|}
\hline Test variable & Variable description & Function \\
\hline CERAD-NAB word list-encoding & $\begin{array}{l}\text { Total number of correctly learned words across } \\
\text { three learning trials (number of words per } \\
\text { trial =10). }\end{array}$ & Verbal episodic learning \\
\hline $\begin{array}{l}\text { CERAD-NAB word list-delayed free } \\
\text { recall }\end{array}$ & $\begin{array}{l}\text { Total number of correctly remembered words } \\
\text { after a delay following encoding. }\end{array}$ & Verbal episodic memory \\
\hline CERAD-NAB word list-savings & $\begin{array}{l}\text { Proportion of correctly recalled words during } \\
\text { delayed free recall relative to words learned } \\
\text { in learning trial } 3 .\end{array}$ & Verbal episodic memory \\
\hline $\begin{array}{l}\text { CERAD-NAB word } \\
\text { list-discriminability }\end{array}$ & $\begin{array}{l}\text { Percent of correctly recognized words from } \\
\text { encoding. }\end{array}$ & Verbal episodic memory \\
\hline $\begin{array}{l}\text { CERAD-NAB word list-intrusion } \\
\text { errors }\end{array}$ & $\begin{array}{l}\text { Total number of intrusions committed during } \\
\text { word list-encoding and delayed free recall. }\end{array}$ & Executive functions \\
\hline CERAD-NAB figures-copy & $\begin{array}{l}\text { Copy of four figures (circle, diamond, } \\
\text { overlapping rectangles, cube). }\end{array}$ & Visuospatial ability \\
\hline CERAD-NAB figures-delayed recall & $\begin{array}{l}\text { Number of correctly reproduced figures from } \\
\text { figures-copy following a delay. }\end{array}$ & Visual memory \\
\hline CERAD-NAB figures-savings & $\begin{array}{l}\text { Proportion correctly reproduced figures at } \\
\text { figures-delayed recall relative to } \\
\text { figures-copy. }\end{array}$ & Visual memory \\
\hline Semantic fluency-animals & Number of animals produced within 1 minute. & Semantic memory \\
\hline Trail making test $\mathrm{A}$ & $\begin{array}{l}\text { Time required to connect circles numbered } \\
\text { from } 1 \text { to } 25 \text { in ascending order. }\end{array}$ & Psychomotor speed \\
\hline Boston naming test (15-items) & $\begin{array}{l}\text { Number of spontaneously correctly named } \\
\text { black and white line drawings } \\
\text { (maximum =15). }\end{array}$ & Language \\
\hline Phonemic fluency & $\begin{array}{l}\text { Number of words produced starting with the } \\
\text { letter } S \text { within } 1 \text { minute. }\end{array}$ & Executive functions \\
\hline Trail making test $\mathrm{B}$ & $\begin{array}{l}\text { Time required to connect circles containing } \\
\text { numbers (1-13) and letters (A-L) in } \\
\text { ascending and alternating order. }\end{array}$ & Executive functions \\
\hline Mean IQCODE & $\begin{array}{l}\text { Mean rating on the } 16 \text {-item informant } \\
\text { questionnaire on perceived changes in } \\
\text { cognitive abilities in daily living during the } \\
\text { preceding 2-years. }\end{array}$ & Informant report \\
\hline
\end{tabular}

CERAD-NAB, Consortium to Establish a Registry for Alzheimer's Disease - Neuropsychological Assessment Battery [31]; IQCODE, Informant Questionnaire on Cognitive Decline in the Elderly [19].

the fitted mixed effects model) were calculated at every two years preceding the MCI diagnosis, corresponding roughly to the timepoints of each visit, for every visit with minimally two NC-MCI datapoints, i.e., until -12 years (cf. Table 2). Additionally, 95\% confidence interval (CI) bands were calculated for the NC-NC and NC-MCI groups, enabling the determination of the timepoints at which estimated NC-NC and NC-MCI functioning diverged. NC-NC and NC-MCI group differences were defined as the timepoint at which the 95\% CIs no longer overlapped. If the $95 \%$ pointwise CIs diverged within the linear interpolated interval of a spline (i.e., between visits), differences were defined as occurring at the following measurement timepoint (i.e., next visit); these timepoints are indicated with vertical lines in the results' graphs. All statistical analyses were performed with SPSS version 21 (SPSS Inc. IBM company, 2012).

\section{RESULTS}

Results of the linear mixed effects models are shown as a cubic spline with $95 \%$ CIs (see Figs. 1 and 2). Please note that the cubic splines and the CIs were computed every two years such that the curves appear less smooth. Additionally, Supplementary Figures 1 and 2 provide information about the distribution of the neuropsychological data and the fitted spline.

\section{Longitudinal courses of episodic and semantic memory performance}

NC-NC verbal encoding performance (Fig. 1A) increased over the 12-year observation period, whereas NC-MCI performance initially increased and then declined approximately four years prior to the $\mathrm{MCI}$ diagnosis. Strikingly, NC-NC and NC-MCI verbal 

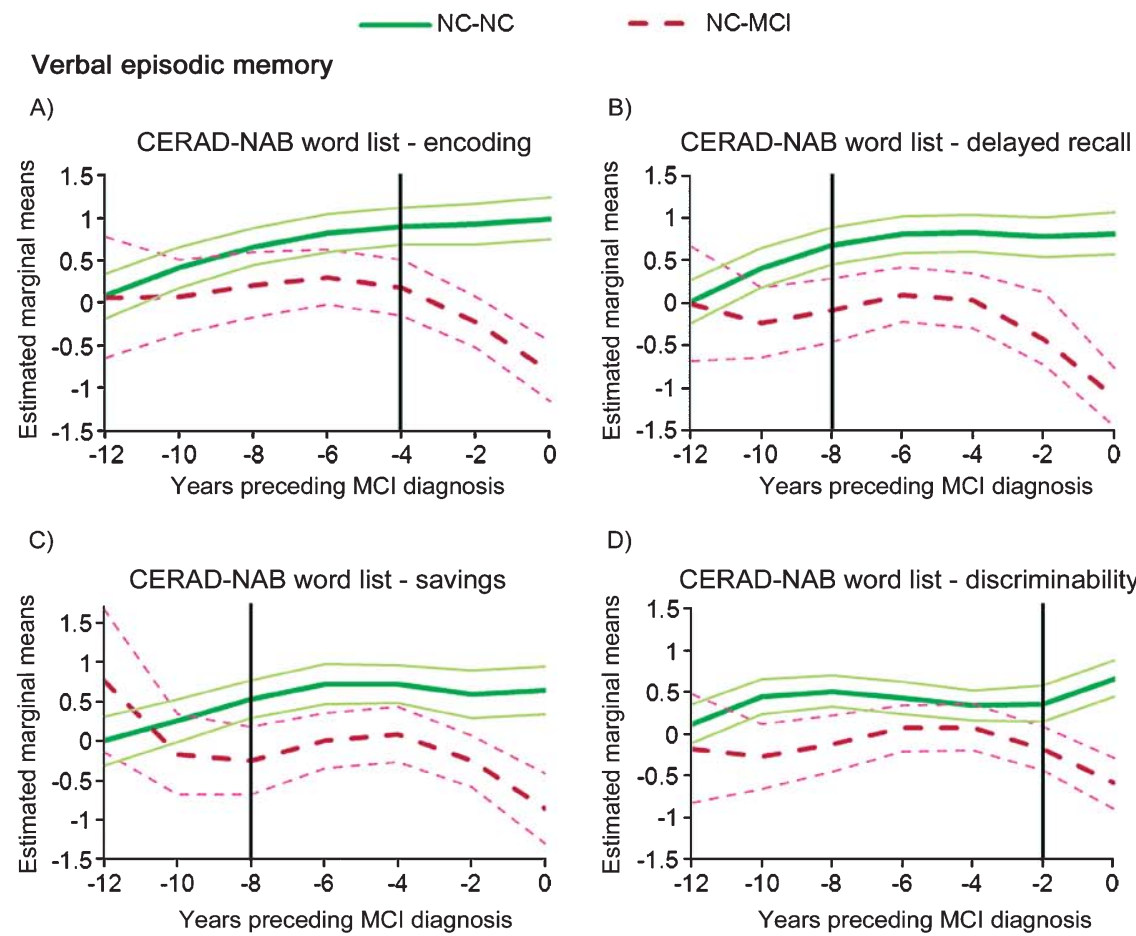

D)

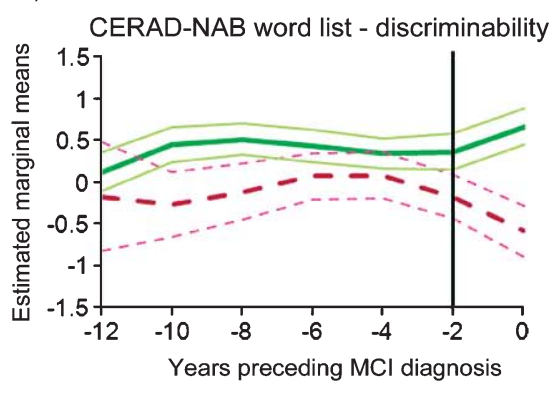

\section{Visual episodic memory}

E)

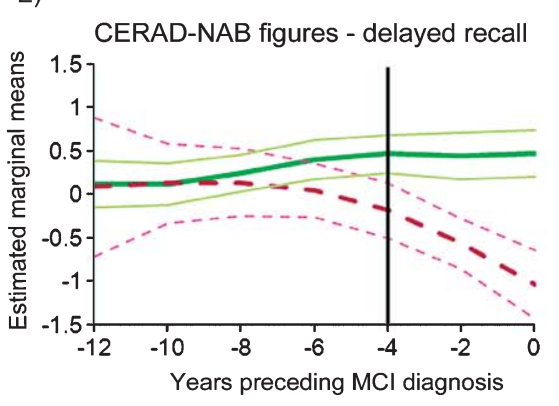

F)

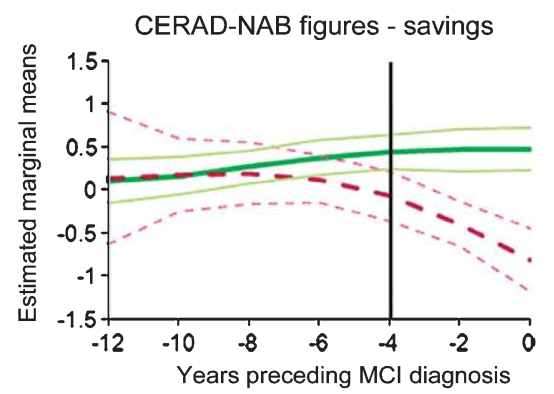

Semantic memory

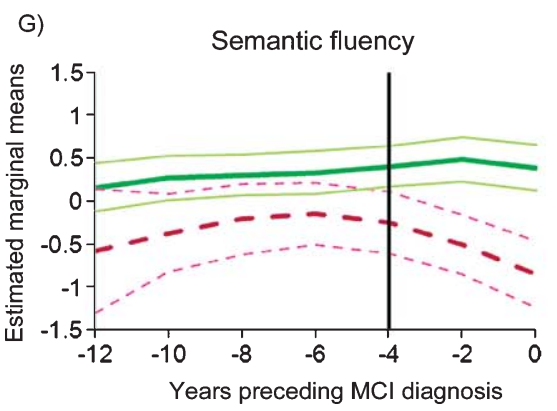

Fig. 1. Cubic splines estimated from linear mixed effects models (with 95\% confidence intervals) of the longitudinal courses of NC-NC and NC-MCI's verbal and visual episodic and semantic memory functioning during the 12 years preceding the diagnosis of MCI due to AD. Cubic splines were computed for data at each observation visit, i.e., every two years preceding the MCI diagnosis. The vertical solid lines indicate the estimated timepoints at which the NC-NC and NC-MCI groups differed. NC-NC, cognitively healthy participants who remained healthy throughout the entire observation period; NC-MCI, initially healthy participants who progressed to MCI; MCI, mild cognitive impairment; CERAD-NAB, Consortium to Establish a Registry for Alzheimer's Disease - Neuropsychological Assessment Battery [31]. 


$$
\text { - NC-MCl }
$$

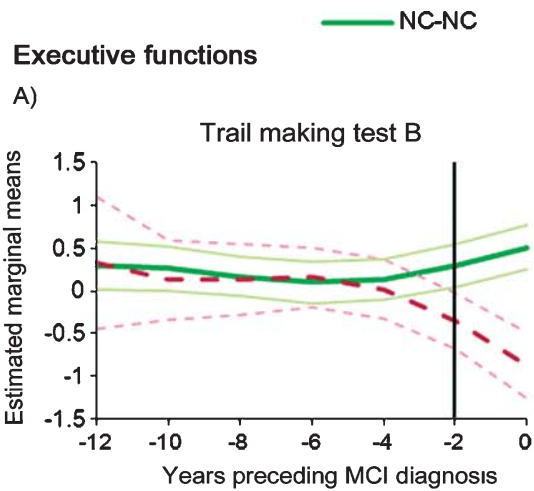

\section{Executive functions}

A)

CERAD-NAB wordlist - intrusion errors

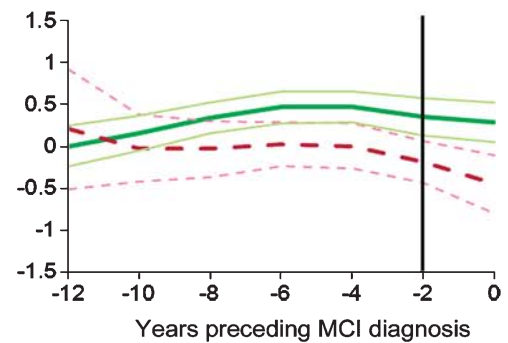

Constructional praxis

D)

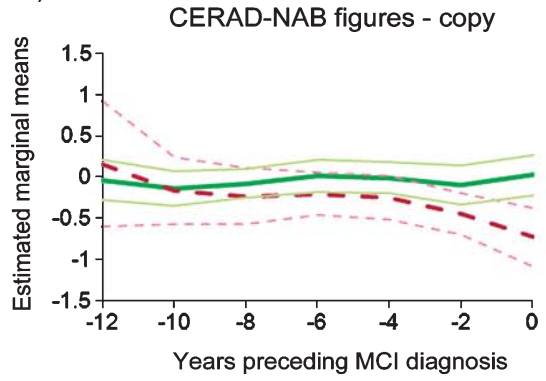

Language

F)

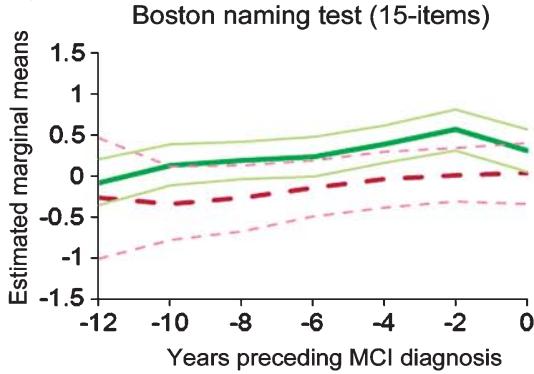

B)

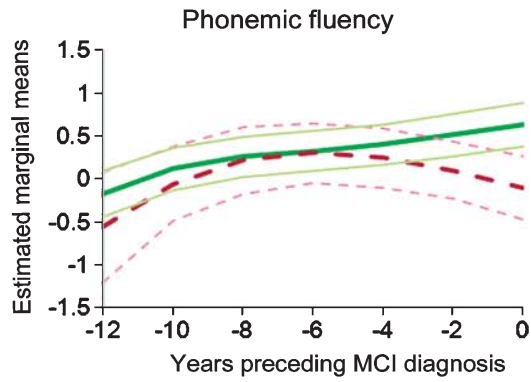

Psychomotor speed

E)

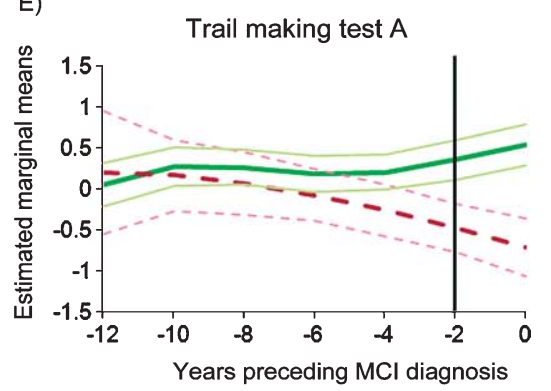

Informant-based report

G)

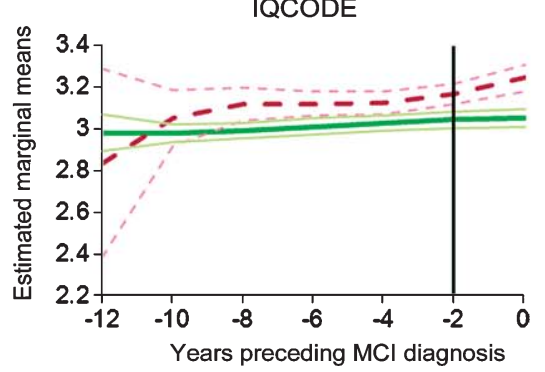

Fig. 2. Cubic splines estimated from linear mixed effects models (with $95 \%$ confidence intervals) of the longitudinal courses of NC-NC and NC-MCI's neuropsychological functioning and informant-based report of cognitive functioning during the 12 years preceding the diagnosis of MCI due to AD. Cubic splines were computed for data at each observation visit, i.e., every two years preceding the MCI diagnosis. The vertical solid lines indicate the estimated timepoints at which the NC-NC and NC-MCI groups differed. NC-NC, cognitively healthy participants who remained healthy throughout the entire observation period; NC-MCI, initially healthy participants who progressed to MCI; MCI, mild cognitive impairment; CERAD-NAB, Consortium to Establish a Registry for Alzheimer's Disease - Neuropsychological Assessment Battery [31]; IQCODE, Informant Questionnaire on Cognitive Decline in the Elderly [19]. 
delayed recall performance diverged already eight years prior to the MCI diagnosis (Fig. 1B). Groups' verbal savings' performance (Fig. 1C) showed a similar evolution, with divergence circa eight years prior to diagnosis. While the NC-MCI group consistently scored lower on verbal discriminability than the NC-NC group, a group difference emerged only approximately two years prior to the MCI diagnosis (Fig. 1D). NC-MCI visual episodic delayed recall and savings performance declined circa four years prior to the MCI diagnosis (Fig. 1E, 1F).

NC-NC and NC-MCI semantic memory functioning (Fig. 1G) differed circa four years prior to the MCI diagnosis.

\section{Longitudinal courses of executive functions}

The NC-NC and NC-MCI groups' TMT-B (Fig. 2A) performance diverged circa two years prior to the $\mathrm{MCI}$ diagnosis. No differences were found in the estimated courses of phonemic fluency performance until the MCI diagnosis (Fig. 2B). The courses of the estimated intrusion errors (Fig. 2C) were similar in both groups and diverged circa two years prior to the MCI diagnosis.

\section{Longitudinal courses of constructional praxis, psychomotor speed, and language performance}

While NC-MCI constructional praxis functioning gradually declined circa six years before the MCI diagnosis, there was no difference between groups (Fig. 2D).

NC-MCI TMT-A performance diverged from the NC-NC group at about -2 years (Fig. 2E), while the modeled courses of NC-NC and NC-MCI 15item Boston naming test performance (Fig. 2F) were comparable.

\section{Longitudinal course of the informant-based report}

The courses of IQCODE scores (Fig. 2G) were similar in both groups, diverging at approximately 2 years. Post-hoc analyses with independent samples' Wilcoxon rank sum-tests revealed these differences to be driven by lower NC-MCI ratings on the following items (all uncorrected $p$-values <0.05): 'remembering things that have happened recently', 'remembering where things are usually kept', 'remembering where to find things which have been put in a different place from usual', and 'using his/her intelligence to understand what is going on and to reason things through'.
At visit 0 (timepoint of MCI diagnosis), the following additional four items significantly differed over groups: 'recalling conversations a few days later', 'remembering what day and month it is', 'learning to use a new gadget or machine around the house', and 'learning new things in general'.

\section{DISCUSSION}

A decline in neuropsychological test performance was detected circa eight years before the diagnosis of MCI due to $\mathrm{AD}$, corresponding to approximately 10.5 years preceding the clinical diagnosis of $\mathrm{AD}$. As expected, group difference in verbal episodic delayed free recall performance was detected first, followed by verbal episodic learning and visual episodic memory four years preceding the MCI diagnosis. A group difference in semantic memory functioning as measured by the 1-minute animal fluency test also emerged four years preceding the MCI diagnosis, whereas changes in executive functions, reduced perceptual speed, and informant-based reports of cognitive decline were apparent two years prior to the MCI diagnosis. Constructional praxis and language functioning as measured by the 15-item Boston naming test remained intact throughout the observation period. Thus, while NC-MCI participants' performance remained largely within normal limits $(z \geq-1.28)$ during the preclinical phase of MCI due to $\mathrm{AD}$, the present group differences indicate that this prodromal phase is characterized by an 8-year progressive decline in objective neuropsychological functioning (see Fig. 3).

The expected early detection of group difference in verbal episodic delayed free recall, savings (circa -8 years), and learning (circa -4 years) performance is in line with the early cortical deposition of neurofibrillary pathology in the entorhinal cortex and hippocampus $[10,11]$, structures associated with the learning and subsequent recall of episodic events [37]. Similarly, previous studies demonstrated that encoding [6, 38], verbal delayed free recall $[5,6,39]$, and verbal percent saving scores [40] were the first functions to decline in the preclinical phase of $\mathrm{AD}$, up to 10 years prior to the clinical diagnosis.

Group difference in visual episodic memory functioning was measurable circa four years prior to the MCI diagnosis (see also [38]). These findings are consistent with volumetric studies demonstrating greater left compared to right medial temporal lobe atrophy in MCI patients who progressed to AD [41]. An additional factor explaining this finding may be that the 


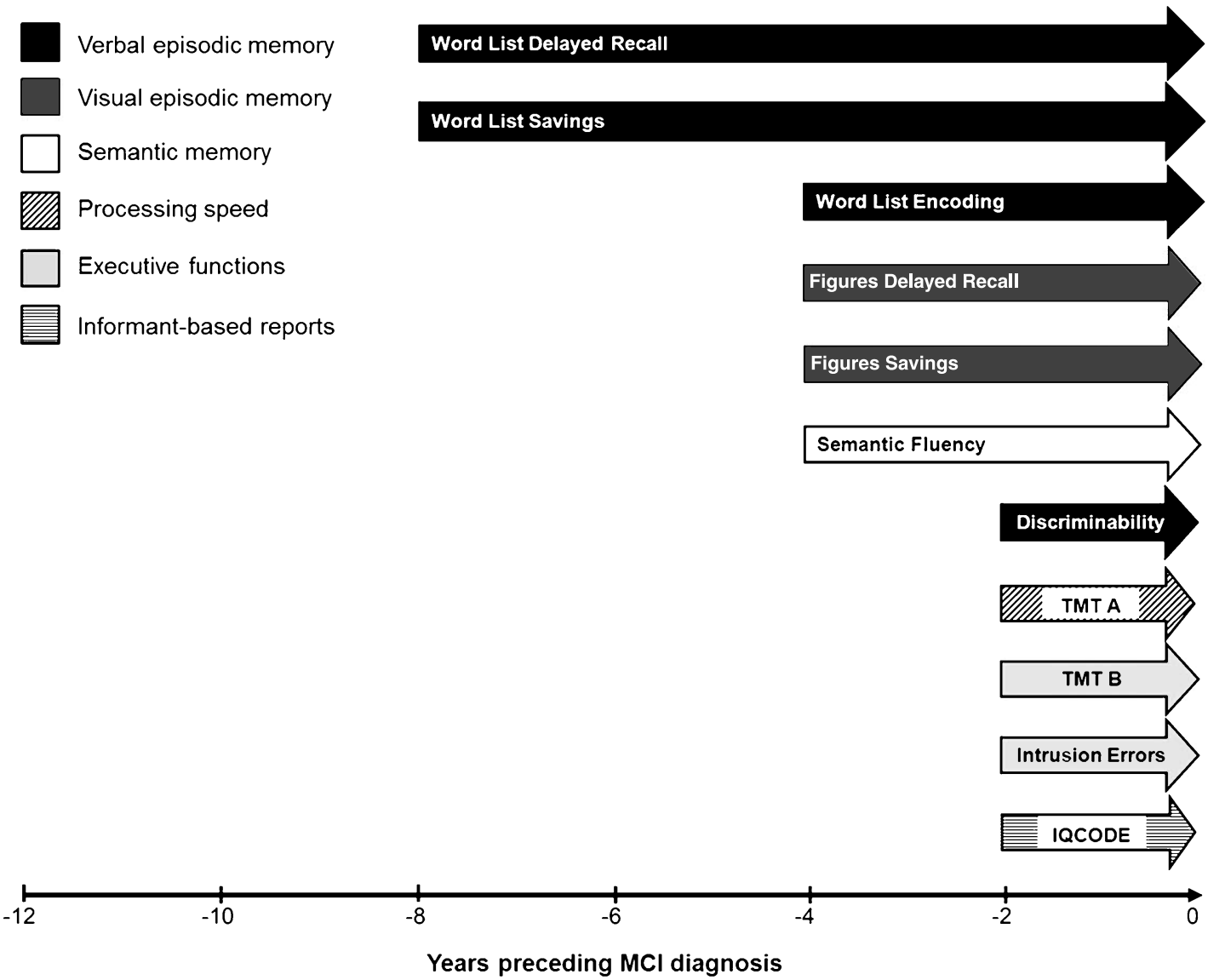

Fig. 3. Temporal order of different neuropsychological declines in the preclinical phase of MCI due to AD. MCI, mild cognitive impairment. Vertical bars indicate first (approximate) timepoints at which performance of future MCI patients diverged from participants who remained cognitively healthy.

visual episodic memory task used here is less demanding than the verbal memory task. Indeed, other studies reported simultaneous verbal and visual episodic memory decline in future AD patients when more complex visual tasks were administered (see, e.g., [6]). Since the use of demographically-corrected $z$-scores does not necessarily uncouple the dependence of test scores on test difficulty, neuropsychological test findings must always be interpreted within the context of the specific tests administered (cf. [35]).

Group difference of semantic memory performance as measured by the 1-minute animal fluency task was found about four years before the MCI diagnosis, circa four years after verbal episodic memory decline (see also [5, 6]). At first blush, this finding appears inconsistent with the earliest cortical neurofibrillary pathology in the perirhinal cortex associated with semantic object memories $[12,13]$. However, the nature of the animal fluency task may account for the present negative finding: it is multicomponential in nature, engaging in addition to semantic memory not only episodic memory, but also language and executive functions [42-44], thereby decreasing its sensitivity to the kind of complex semantic object memory impairments predicted to follow perirhinal cortex damage [45]. Thus, more sensitive tests of confusable semantic object processing are required to more specifically measure perirhinal cortex dysfunction [13]. Alternatively, the assessment of semantic processing in several different categories may provide a more robust and sensitive measure of semantic memory performance. Indeed, Amieva et al. [14] examined the longitudinal courses of cognitive functioning in preclinical AD patients and demographically matched healthy control participants over a 14-year period and found that semantic memory as assessed with a total score on four semantic fluency tasks (colors, animals, fruits, cities) declined 12 years prior to the clinical diagnosis of $\mathrm{AD}$, three years preceding the occurrence of visual episodic memory decline. 
Degeneration of the temporal-frontal projection systems occurs in $\mathrm{AD}$ [46], presumably resulting in executive dysfunction such as poor planning and sequencing, response inhibition, spontaneous flexibility, or set shifting [7]. Executive dysfunction in MCI patients typically follows memory impairments by circa four years [4-6]. Our results are consistent with these findings: executive functions (TMT-B, intrusion errors) declined approximately 2 and 4.5 years prior to the present $\mathrm{MCI}$ and $\mathrm{AD}$ diagnoses, respectively, 6 years following verbal episodic memory decline. These findings corroborate the hypothesis that not only patients with single domain amnestic MCI, but also MCI patients with additional executive dysfunction, have a heightened risk to progress to AD [47].

Constructional praxis and simple confrontation naming abilities appear to be relatively spared in the preclinical stage of MCI $[48,49]$. We note that although these functions were not affected in the present NCMCI sample, more sensitive tests may reveal differences between healthy individuals and future AD patients at the time of MCI diagnosis (see, e.g., [50]).

The present findings support the claim that the earliest cognitive changes in future dementia patients may be perceived by family members before cognitive impairments manifest themselves in daily life [18]. As informants were required to assess patients' cognitive functioning during the preceding two years, the present finding suggests that informants already perceived changes in patients' cognitive functioning between two and four years prior to the MCI diagnosis. Post-hoc analyses revealed that this finding was primarily driven by items associated with episodic memory functioning. Thus, informant-based reports such as the IQCODE may indicate future progression to dementia already in the preclinical stage of MCI, when activities of daily living are still within normal ranges.

The dynamic biomarker model proposed by Jack et al. [51] hypothesizes that markers of amyloid- $\beta$ pathology (e.g., CSF, PET imaging) become abnormal first in preclinical $\mathrm{AD}$, followed by markers of neurodegeneration (i.e., CSF tau protein, hypometabolism on fluorodeoxyglucose PET, and atrophy on structural MRI). Cognitive impairments are hypothesized to emerge last, shortly prior to the diagnosis. While a number of studies corroborate the temporal order of amyloid- $\beta$, neurodegenerative and cognitive abnormalities in future AD patients (see [52]), these studies typically used global cognitive measures (e.g., MMSE total score), which are necessarily less specific to cognitive impairments associated with anteromedial temporal lobe neurofibrillary pathology than episodic memory tests, for example. Indeed, more sensitive neuropsychological measures such as verbal learning und delayed recall indices detect brain pathology quite early in the prodromal phase, potentially as early as ADrelated CSF and imaging biomarkers (for example, see [39]). In this context, the sensitivity and feasibility (non-invasive, accessible, and relatively inexpensive) of episodic memory testing for case-finding or screening efforts are evident. Clearly, a full clinical dementia workup is ideally comprised of all the interdisciplinary measures noted above to maximize diagnostic accuracy.

The present results should be replicated in a larger sample. Moreover, the sample characteristics should be taken into consideration when appraising the generalizability of the present findings: the samples used in this study were convenience subsamples emanating from the original Basel Study initiated in 1959. These subsamples predominantly consisted of (former) employees of the pharmaceutical industry in Basel [53]. We also note that our methodological approach is descriptive in nature as results were based on $95 \%$-confidence bands indicating the range within which true values are likely to be located. Since confidence intervals depend on the samplesize, where largersamples lead to more confident results and narrower confidence intervals, the timepoints at which cognitive functioning in the NC-NC and NC-MCI groups actually diverge are most likely earlier than reported here (see also [14]). Given the relatively small sample sizes, the precise timepoints at which each cognitive function declines in the NC-MCI group (e.g., -8 years, -4 years) must be interpreted with caution. Specifically, the present results should be interpreted as a sequence of events rather than exact timepoints of incident cognitive changes. Additionally, we note that tests with a restricted range of possible scores (i.e., word list intrusion errors, figures - copy) typically result in nonnormal distributions, such that the curve estimates of these performance measures should be interpreted with caution. Finally, some subtests of the CERAD-NAB may not be sensitive enough to detect actual cognitive decline in NC-MCI participants primarily because of ceiling effects. Although the results of the present study clearly indicate that differences between the NC$\mathrm{NC}$ and NC-MCI groups emerge many years before a clinical diagnosis of $\mathrm{MCI}$, the direct applicability of this finding to clinical practice remains limited. By diagnostic definition, the cognitive performance of the NC-MCI group was within normal limits until shortly before the MCI diagnosis. To circumvent this issue and enable the present results to be implemented in the clinical assessment of individual patients, normative baseline data of individuals that have verifiably 
remained cognitively healthy for several years must be obtained, and/or the assessed individual should be followed over time with neuropsychological testing. Future research should determine whether change scores on neuropsychological tests (see, for example, $[54,55])$ provide more sensitive markers of future MCI due to $\mathrm{AD}$ than cross-sectional scores, and the optimal combination of neuropsychological test scores and CSF, PET, and structural MRI measures to predict progression to AD dementia.

The present findings provide evidence for a long preclinical stage of MCI due to AD characterized by declining cognitive functioning of particularly verbal episodic memory. Visual episodic memory and semantic memory declined later, followed by a decline in executive functions approximately two years preceding the MCI diagnosis. We note that the NC-MCI group's visuospatial abilities and simple confrontation naming remained comparable to the NC-NC group throughout the observation period. Reduced verbal episodic delayed recall performance eight years preceding the MCI diagnosis corresponds to the timepoint at which some studies first detect abnormal CSF and imaging biomarkers [39]. Thus, our results indicate that cognitive markers have the potential to identify future $\mathrm{AD}$ patients at the earliest stages of MCI due to $\mathrm{AD}$, providing the opportunity to better understand the pathogenesis of $\mathrm{AD}$ as well as initiate treatment interventions.

\section{ACKNOWLEDGMENTS}

This work was supported by grants from the Swiss National Science Foundation (grant numbers 3200-049107 to A.U.M. and PZ00P1_126493 to K.I.T.), from the Novartis Foundation, Basel Switzerland. (A.U.M.), and the Alfred and Anneliese Sutter-Stöttner Stiftung (A.U.M.). We gratefully acknowledge the continuous enthusiasm of the participants of the Basel Study on the Elderly project.

Authors' disclosures available online (http://j-alz. com/manuscript-disclosures/15-0137r2).

\section{SUPPLEMENTARY MATERIAL}

The supplementary material is available in the electronic version of this article: http://dx.doi.org/ 10.3233/JAD-150137.

\section{REFERENCES}

[1] Duara R, Loewenstein DA, Greig MT, Potter E, Barker W, Raj A, Schinka J, Borenstein A, Schoenberg M, Wu Y, Banko
J, Potter H (2011) Pre-MCI and MCI: Neuropsychological, clinical, and imaging features and progression rates. Am J Geriatr Psychiatry 19, 951-960.

[2] Caselli RJ, Reiman EM, Locke DC, Hutton ML, Hentz JG, Hoffman-Snyder C, Woodruff BK, Alexander GE, Osborne D (2007) Cognitive domain decline in healthy apolipoprotein $\mathrm{E} \varepsilon 4$ homozygotes before the diagnosis of mild cognitive impairment. Arch Neurol 64, 1306-1311.

[3] Mungas D, Beckett L, Harvey D, Tomaszewski Farias D, Reed B, Carmichael O, Olichney J, Miller J, DeCarli C (2010) Heterogeneity of cognitive trajectories in diverse older persons. Psychol Aging 25, 606-619.

[4] Albert MS, Blacker D, Moss MB, Tanzi R, McArdle JJ (2007) Longitudinal change in cognitive performance among individuals with mild cognitive impairment. Neuropsychology $\mathbf{2 1}$, 158-169.

[5] Grober E, Hall CB, Lipton RB, Zonderman AB, Resnick SM, Kawas C (2008) Memory impairment, executive dysfunction, and intellectual decline in preclinical Alzheimer's disease. J Int Neuropsychol Soc 14, 266-278.

[6] Saxton J, Lopez OL, Ratcliff G, Dulberg C, Fried LP, Carlson MC, Newman AB, Kuller L (2004) Preclinical Alzheimer disease: Neuropsychological test performance 1.5 to 8 years prior to onset. Neurology 63, 2341-2347.

[7] Salmon DP (2011) Neuropsychological features of mild cognitive impairment and preclinical Alzheimer's disease. Curr Top Behav Neurosci 10, 187-212.

[8] Hall CB, Lipton RB, Sliwinski M, Stewart WF (2000) A change point model for estimating the onset of cognitive decline in preclinical Alzheimer's disease. Stat Med 19, 15551566.

[9] Grober E, Dickson D, Sliwinski MJ, Buschke H, Katz M, Crystal H, Lipton RB (1999) Memory and mental status correlates of modified Braak staging. Neurobiol Aging 20, 573-579.

[10] Braak H, Braak E (1991) Neuropathological stageing of Alzheimer-related changes. Acta Neuropathol (Berl) 82, 239259.

[11] Lipton PA, Eichenbaum H (2008) Complementary roles of hippocampus and medial entorhinal cortex in episodic memory. Neural Plast 2008, e258467.

[12] Taylor KI, Probst A (2008) Anatomic localization of the transentorhinal region of the perirhinal cortex. Neurobiol Aging 29, 1591-1596.

[13] Kivisaari SL, Tyler LK, Monsch AU, Taylor KI (2012) Medial perirhinal cortex disambiguates confusable objects. Brain 135, 3757-3769.

[14] Amieva H, Le Goff M, Millet X, Orgogozo J-M, Pérès K, Barberger-Gateau P, Jacqmin-Gadda H, Dartigues J-F (2008) Prodromal Alzheimer's disease: Successive emergence of the clinical symptoms. Ann Neurol 64, 492-498.

[15] Howieson DB, Carlson NE, Moore MM, Wasserman D, Abendroth CD, Payne-Murphy J, Kaye JA (2008) Trajectory of mild cognitive impairment onset. J Int Neuropsychol Soc 14, 192-198.

[16] Twamley EW, Ropacki SA, Bondi MW (2006) Neuropsychological and neuroimaging changes in preclinical Alzheimer's disease. J Int Neuropsychol Soc 12, 707-735.

[17] Carr DB, Gray S, Baty J, Morris JC (2000) The value of informant versus individual's complaints of memory impairment in early dementia. Neurology 55, 1724-1726.

[18] Gifford KA, Liu D, Lu Z, Tripodis Y, Cantwell NG, Palmisano J, Kowall N, Jefferson AL (2014) The source of cognitive complaints predicts diagnostic conversion differentially among nondemented older adults. Alzheimers Dement 10, 319-327. 
[19] Isella V, Villa L, Russo A, Regazzoni R, Ferrarese C, Appollonio IM (2006) Discriminative and predictive power of an informant report in mild cognitive impairment. $J$ Neurol Neurosurg Psychiatry 77, 166-171.

[20] Jorm AF, Korten AE (1988) Assessment of cognitive decline in the elderly by informant interview. Br J Psychiatry 152, 209-213.

[21] Ehrensperger MM, Berres M, Taylor KI, Monsch AU (2010) Screening properties of the German IQCODE with a twoyear time frame in MCI and early Alzheimer's disease. Int Psychogeriatr 22, 91-100.

[22] Storandt M, Grant EA, Miller J, Morris JC (2006) Longitudinal course and neuropathologic outcomes in original vs revised MCI and in pre-MCI. Neurology 67, 467-473.

[23] Wilson RS, Leurgans SE, Boyle PA, Bennett DA (2011) Cognitive decline in prodromal Alzheimer disease and mild cognitive impairment. Arch Neurol 68, 351-356.

[24] Schmid NS, Taylor KI, Foldi NS, Berres M, Monsch AU (2013) Neuropsychological signs of Alzheimer's disease 8 years prior to diagnosis. J Alzheimers Dis 34, 537-546.

[25] Winblad B, Palmer K, Kivipelto M, Jelic V, Fratiglioni L, Wahlund L-O, Nordberg A, Bäckman L, Albert M, Almkvist $\mathrm{O}$, Arai H, Basun H, Blennow K, De Leon M, DeCarli C, Erkinjuntti T, Giacobini E, Graff C, Hardy J, Jack C, Jorm A, Ritchie K, Van Duijn C, Visser P, Petersen RC (2004) Mild cognitive impairment - beyond controversies, towards a consensus: Report of the international working group on mild cognitive impairment. J Intern Med 256, 240-246.

[26] Petersen RC (2004) Mild cognitive impairment as a diagnostic entity. J Intern Med 256, 183-194.

[27] Albert MS, DeKosky ST, Dickson D, Dubois B, Feldman HH, Fox NC, Gamst A, Holtzman DM, Jagust WJ, Petersen RC, Snyder PJ, Carrillo MC, Thies B, Phelps CH (2011) The diagnosis of mild cognitive impairment due to Alzheimer's disease: Recommendations from the National Institute on Aging-Alzheimer's Association workgroups on diagnostic guidelines for Alzheimer's disease. Alzheimers Dement 7 , 270-279.

[28] American Psychiatric Association (1994) Diagnostic and statistical manual of mental disorders: DSM-IV, American Psychiatric Association Press, Washington, DC.

[29] McKhann G, Drachman D, Folstein M, Katzman R, Price D, Stadlan EM (1984) Clinical diagnosis of Alzheimer's disease report of the NINCDS-ADRDA Work Group under the auspices of Department of Health and Human Services Task Force on Alzheimer's Disease. Neurology 34, 939-944.

[30] Folstein MF, Folstein SE, McHugh PR (1975) "Mini-mental state": A practical method for grading the cognitive state of patients for the clinician. J Psychiatr Res 12, 189-198.

[31] Morris JC, Mohs RC, Rogers H, Fillenbaum G, Heyman A (1988) Consortium to Establish a Registry for Alzheimer's disease (CERAD) and neuropsychological assessment of Alzheimer's disease. Psychopharmalogical Bull 24, 641-652.

[32] Berres M, Zehnder A, Bläsi S, Monsch AU (2008) Evaluation of diagnostic scores with adjustment for covariates. Stat Med 27, 1777-1790.

[33] Berres M, Monsch AU, Bernasconi F, Thalmann B, Stähelin HB (2000) Normal ranges of neuropsychological tests for the disease of Alzheimer's disease. Stud Health Technol Inform 77, 195-199.

[34] Delis DC, Kramer JH, Kaplan E, Ober BA (1987) California Verbal Learning Test, Psychological Corporation, San Antonio, TX.
[35] Beck IR, Gagneux-Zurbriggen A, Berres M, Taylor KI, Monsch AU (2012) Comparison of verbal episodic memory measures: Consortium to establish a registry for Alzheimer's disease - Neuropsychological assessment battery (CERADNAB) versus California verbal learning test (CVLT). Arch Clin Neuropsychol 27, 510-519.

[36] Schmid NS, Ehrensperger MM, Berres M, Beck IR, Monsch AU (2014) The extension of the German CERADNeuropsychological assessment battery with tests assessing subcortical, executive and frontal functions improves accuracy in dementia diagnosis. Dement Geriatr Cogn Extra 4, 322-334.

[37] Aggleton JP, Brown MW (1999) Episodic memory, amnesia, and the hippocampal-anterior thalamic axis. Behav Brain Sci 22, 425-444.

[38] Bilgel M, An Y, Lang A, Prince J, Ferrucci L, Jedynak B, Resnick SM (2014) Trajectories of Alzheimer disease-related cognitive measures in a longitudinal sample. Alzheimers Dement 10, 735-742.e4.

[39] Jedynak BM, Lang A, Liu B, Katz E, Zhang Y, Wyman BT, Raunig D, Jedynak CP, Caffo B, Prince JL (2012) A computational neurodegenerative disease progression score: Method and results with the Alzheimer's disease neuroimaging initiative cohort. Neuroimage 63, 1478-1486.

[40] Libon DJ, Bondi MW, Price CC, Lamar M, Eppig J, Wambach DM, Nieves C, Delano-Wood L, Giovannetti T, Lippa C Kabasakalian A, Cosentino S, Swenson R, Penney DL (2011) Verbal serial list learning in mild cognitive impairment: A profile analysis of interference, forgetting, and errors. J Int Neuropsychol Soc 17, 905-914.

[41] Ferreira LK, Diniz BS, Forlenza OV, Busatto GF, Zanetti MV (2011) Neurostructural predictors of Alzheimer's disease: A meta-analysis of VBM studies. Neurobiol Aging 32, 17331741

[42] Hirni DI, Kivisaari SL, Monsch AU, Taylor KI (2013) Distinct neuroanatomical bases of episodic and semantic memory performance in Alzheimer's disease. Neuropsychologia 51, 930-937.

[43] Henry JD, Crawford JR, Phillips LH (2004) Verbal fluency performance in dementia of the Alzheimer's type: A metaanalysis. Neuropsychologia 42, 1212-1222.

[44] Beck IR, Schmid NS, Berres M, Monsch AU (2014) Establishing robust cognitive dimensions for characterization and differentiation of patients with Alzheimer's disease, mild cognitive impairment, frontotemporal dementia and depression. Int J Geriatr Psychiatry 29, 624-634.

[45] Taylor KI, Moss HE, Tyler LK. (2007) The conceptual structure account: A cognitive model of semantic memory and its neural instantiation. In Neural Basis of Semantic Memory, Hart J, Kraut M, eds. Cambridge University Press, Cambridge, pp. 265-301.

[46] Chen T, Chen Y, Cheng T, Hua M, Liu H, Chiu M (2009) Executive dysfunction and periventricular diffusion tensor changes in amnesic mild cognitive impairment and early Alzheimer's disease. Hum Brain Mapp 30, 3826-3836.

[47] Chen P, Ratcliff G, Belle SH, Cauley JA, DeKosky ST, Ganguli M (2001) Patterns of cognitive decline in presymptomatic Alzheimer disease: A prospective community study. Arch Gen Psychiatry 58, 853-858.

[48] Collie A, Maruff P (2000) The neuropsychology of preclinical Alzheimer's disease and mild cognitive impairment. Neurosci Biobehav Rev 24, 365-374.

[49] Karrasch M, Sinervä E, Grönholm P, Rinne J, Laine M (2005) CERAD test performances in amnestic mild cognitive impairment and Alzheimer's disease. Acta Neurol Scand 111, 172-179. 
[50] Kasai M, Meguro K, Hashimoto R, Ishizaki J, Yamadori A, Mori E (2006) Non-verbal learning is impaired in very mild Alzheimer's disease (CDR 0.5): Normative data from the learning version of the Rey-Osterrieth Complex Figure Test. Psychiatry Clin Neurosci 60, 139-146.

[51] Jack CR, Holtzman DM (2013) Biomarker modeling of Alzheimer's disease. Neuron 80, 1347-1358.

[52] Buchhave P, Minthon L, Zetterberg H, Wallin AK, Blennow K, Hansson O (2012) Cerebrospinal fluid levels of $\beta$-amyloid $1-42$, but not of tau, are fully changed already 5 to 10 years before the onset of Alzheimer dementia. Arch Gen Psychiatry 69, 98-106.
[53] Widmer LK, Stähelin HB, Nissen C, da Silva A (1981) Venen-, Arterien-Krankheiten, koronare Herzkrankheit bei Berufstätigen - Prospektiv-epidemiologische Untersuchung Basler Studie I-III 1959-1978, Hans Huber Verlag, Bern.

[54] Bläsi S, Zehnder A, Berres M, Taylor KI, Spiegel R, Monsch $\mathrm{AU}$ (2009) Norms for change in episodic memory as a prerequisite for the diagnosis of mild cognitive impairment (MCI). Neuropsychology 23, 189-200.

[55] Zehnder AE, Bläsi S, Berres M, Spiegel R, Monsch AU (2007) Lack of practice effects on neuropsychological tests as early cognitive markers of Alzheimer disease? Am J Alzheimers Dis Other Demen 22, 416-426. 